\title{
X-ray Phase-Contrast Radiography and Tomography with a Multiaperture Analyzer
}

\author{
M. Endrizzi ${ }^{1,{ }^{*}}$ F. A. Vittoria, ${ }^{1}$ L. Rigon, ${ }^{2,3}$ D. Dreossi, ${ }^{4}$ F. Iacoviello, ${ }^{5}$ P. R. Shearing, ${ }^{5}$ and A. Olivo ${ }^{1}$ \\ ${ }^{1}$ Department of Medical Physics and Biomedical Engineering, University College London, \\ Gower Street, London WC1E 6BT, United Kingdom \\ ${ }^{2}$ Physics Department, University of Trieste, Via Valerio 2, 34127 Trieste, Italy \\ ${ }^{3}$ Istituto Nazionale di Fisica Nulceare, Sezione di Trieste, Via Valerio 2, 34127 Trieste, Italy \\ ${ }^{4}$ Sincrotrone Trieste SCpA, S.S. 14 km 163.5, 34012 Basovizza Trieste, Italy \\ ${ }^{5}$ Electrochemical Innovation Lab, Department of Chemical Engineering, University College London, WC1E 7JE, United Kingdom
}

(Received 25 January 2017; published 14 June 2017)

\begin{abstract}
We present a multiaperture analyzer setup for performing $\mathrm{x}$-ray phase contrast imaging in planar and three-dimensional modalities. The method is based on strongly structuring the x-ray beam with an amplitude modulator, before it reaches the sample, and on a multiaperture analyzing element before detection. A multislice representation of the sample is used to establish a quantitative relation between projection images and the corresponding three-dimensional distributions, leading to successful tomographic reconstruction. Sample absorption, phase, and scattering are retrieved from the measurement of five intensity projections. The method is tested on custom-built phantoms with synchrotron radiation: sample absorption and phase can be reliably retrieved also in combination with strong scatterers, simultaneously attaining high sensitivity and dynamic range.
\end{abstract}

DOI: 10.1103/PhysRevLett.118.243902

Conventional radiography is based on the attenuation of $\mathrm{x}$ rays for generating contrast. In $\mathrm{X}$-ray phase-contrast imaging (XPCI) [1,2], additional contrast mechanisms, generating from the phase shifts imparted to the beam by the sample, contribute to the image formation process. This can improve the visibility of a large variety of details and its applications span across many different fields, encompassing materials science, security, biology, and medicine. Among various implementations with which it is possible to obtain $\mathrm{x}$-ray phase-contrast images [3-18], we focus here on edge illumination (EI) [19]. EI can provide quantitative attenuation, phase [20] and dark-field [21] representation of a sample, and can be adapted for use with synchrotron radiation, microfocus tubes, and conventional rotating anode sources with extended focal spots [22,23]. It exhibits negligible requirements in terms of temporal or spatial coherence [24,25], provides high sensitivity [26-28], is robust against mechanical and thermal instabilities [29,30], and enables low-dose implementations of XPCI in planar and three-dimensional imaging [31,32].

We introduce here a multiaperture analyzer setup for performing EI in situations where very wide angular ranges must be explored, for example, in the presence of strong scatterers. Through experimental tests on custombuilt phantoms, we show that refraction can be accurately retrieved independently from the presence of large amounts

Published by the American Physical Society under the terms of the Creative Commons Attribution 4.0 International license. Further distribution of this work must maintain attribution to the author(s) and the published article's title, journal citation, and DOI. of scattering. Two spatial resolutions are investigated, showing that the retrieved signals are independent from this parameter. A simple model based on a multislice representation of the sample enables the reconstruction of the three-dimensional images. The method retrieves absorption, refraction, and dark-field images, simultaneously providing high sensitivity and large dynamic range for both planar and three-dimensional XPCI applications. These proof-of-principle experiments are performed using monochromatic synchrotron radiation; however, EI methods can be successfully implemented also using rotating anode X-ray tubes with extended focal spots.

The experimental setup is sketched in Fig. 1(a). The beam from a bending magnet source is monochromatized with a double $\mathrm{Si}$ (111) crystal and then shaped by a narrow aperture, before it impinges on the sample. It is then analyzed by a set of apertures positioned immediately before a digital detector. In order to achieve different levels of illumination, the detector and the detector apertures are scanned along the $y$ direction. The data collected with each detector exposure result in a single line of pixels, and a 2D image is built by scanning the sample along the $y$ direction and exposing multiple times.

The illumination function (IF) describes how the detected intensity varies depending on the relative displacement $\bar{y}$ between presample and detector apertures. By using a geometrical optics model, the intensity measured at the detector is described as a convolution between the IF $L(\bar{y})$ and the object function $O(x, y)$ :

$$
I(\bar{y})=\int L(\bar{y}-y) O(y) d y .
$$




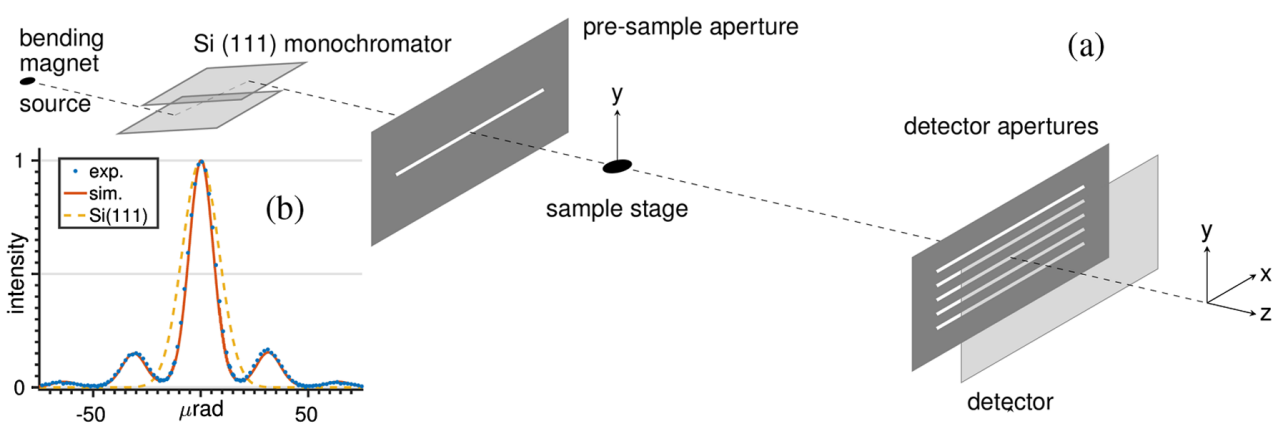

FIG. 1. Experimental setup (a): monochromatic synchrotron radiation is shaped to a narrow beam by a simple aperture before the sample. It is then analyzed by a set of apertures before detection. 2D images are built by scanning the sample along $y$. Simulated (solid line) and experimental (circles) illumination functions (b): an approximate Si (111) rocking curve is also plotted (dashed line) for comparison $(20 \mathrm{keV})$.

Transmission, refraction, and scattering properties of the sample were retrieved by using a multi-Gaussian model for the IF [21]:

$$
I(\bar{y})=\sum_{m} \sum_{n} A_{m n} \exp \left(-\frac{\left(\bar{y}-\mu_{m n}\right)^{2}}{2 \sigma_{m n}^{2}}\right)
$$

where $L(\bar{y})=\sum_{n}\left(A_{n} / \sqrt{2 \pi \sigma_{n}^{2}}\right) \exp \left[-\left(\bar{y}-\mu_{n}\right)^{2} / 2 \sigma_{n}^{2}\right],(n=$ $1 \ldots N)$, and $O(\bar{y})=\sum_{m}\left(A_{m} / \sqrt{2 \pi \sigma_{m}^{2}}\right) \exp \left[-\left(\bar{y}-\mu_{m}\right)^{2} /\right.$ $\left.2 \sigma_{m}^{2}\right], \quad(m=1 \ldots M)$. The parameters are defined as follows: $\mu_{m n}=\mu_{m}+\mu_{n}, \sigma_{m n}^{2}=\sigma_{m}^{2}+\sigma_{n}^{2}$, and $A_{m n}=A_{m} A_{n}$ $\left(1 / \sqrt{2 \pi \sigma_{m n}^{2}}\right)$. We note that beam absorption and refraction can be both included in the object function $O(y)$ as a multiplicative factor $t$ and a shift $\Delta y_{R}$ of the center of the distribution, respectively. In this formulation, a purely absorbing object is represented by a Dirac's delta function centered in zero and multiplied by a factor $t$, which indicates the transmitted to incident intensity ratio. If the sample is also refracting, the delta function is shifted $O(y)=t \delta\left(y-\Delta y_{R}\right)$; thus, the effect on the IF is a reduction in the transmitted intensity by the factor $t$ plus a $\Delta y_{R}$ shift due to refraction. In the case of a sample exhibiting also dark-field contrast, the object function $O(y)$ has a finite width and the presence of the sample results in a broadened IF.

Phase images can be obtained by numerical integration of the refraction images by taking into account that the angle $\alpha=(\lambda / 2 \pi) \partial_{y} \Phi(x, y)$ is proportional to the gradient of the object's phase shift $\Phi(x, y)$, where $\lambda$ is the wavelength. In the case of a parallel beam geometry, the refraction angle is measured as the ratio between the relative shift of the IF and the sample-to-detector distance $\alpha=\Delta y_{R} / z_{s d}$.

A thick sample can be represented with a multislice approach, by separately considering the subsequent effect of thin sections along the beam axis. We define $O_{k}$ a thin but finite thickness $\Delta_{z}$ section of the object along $z$. The entire object is obtained by the sum of its sections (with $k=1 \ldots K)$ and its extent along $z$ is given by $Z_{o}=\Delta_{z} K$. The intensity measured at the detector can then be expressed in terms of the contributions from separate layers

$$
\begin{aligned}
I(\bar{y}) & =(L \otimes O)(\bar{y}) \\
& =\left(L \otimes O_{1} \otimes O_{2} \otimes \ldots \otimes O_{K}\right)(\bar{y}),
\end{aligned}
$$

where the more compact notation $\otimes$ was used for convolution. Let us consider, for simplicity's sake, a singleGaussian model for the object's section functions $O_{k}$. In this case, the entire object function $O(y)=\left(O_{1} \otimes O_{2}\right.$ $\left.\otimes \ldots \otimes O_{K}\right)(y)$ is still a Gaussian, with mean $\mu_{O}=$ $\sum_{1}^{K} \mu_{k}$, variance $\sigma_{O}^{2}=\sum_{1}^{K} \sigma_{k}^{2}$, and amplitude $A_{O}=$ $\left(1 / \sqrt{2 \pi \sigma_{O}^{2}}\right) \prod_{1}^{K} A_{k}$; this is now expressed as a function of the contributions from the individual thin layers. The intensity transmitted by the entire object becomes

$$
t=\prod_{1}^{K} A_{k}=\prod_{1}^{K} e^{-\left(4 \pi \beta_{k} \Delta_{z} / \lambda\right)}
$$

and a similar result is obtained for the refraction

$$
\Delta y_{R}=\sum_{1}^{K} \mu_{k}=-z_{o d} \partial_{y} \sum_{1}^{K} \delta_{k} \Delta_{z}
$$

where $\delta$ is the decrement from unity of the material's refractive index, $\beta$ its imaginary part, and $\Phi_{k}=$ $-(2 \pi / \lambda) \delta_{k} \Delta_{z}$ is the phase shift introduced by the $k$ th object's layer. If the section thickness $\Delta y$ is small enough to allow a transition to the continuous formulation, we obtain

$$
\begin{gathered}
t=e^{-(4 \pi / \lambda) \int \beta(z) d z}, \\
\Delta y_{R}=-z_{o d} \partial_{y} \int \delta(z) d z,
\end{gathered}
$$

and, similarly, for the dark-field signal

$$
\sigma_{O}^{2}=\int \sigma^{2}(z) d z
$$

Along with absorption and phase, also the dark-field signal $\sigma_{O}^{2}$ can be cast as an integral along the beam path. By 
collecting a number of different views while rotating the sample around the $y$ axis, the three-dimensional distribution of the width of the object function $\sigma^{2}(x, y, z)$ can be calculated by means of the inverse radon transform or the filtered back projection algorithms, as is routinely done in computed tomography (CT). Similar expressions, for the behavior of the object's scattering distribution as a function of the object's thickness, were obtained in the context of other approaches for performing x-ray phase-contrast imaging [33-36].

The experiment was performed at the SYRMEP beam line (Elettra Sincrotrone Trieste, Italy). The presample slit was $40 \mathrm{~mm}$ along $x$ and its aperture along $y$ was set at $20 \mu \mathrm{m}$. The detector apertures were $23 \mu \mathrm{m}$ and arranged at a regular period of $79 \mu \mathrm{m}$. The beam energy was $20 \mathrm{keV}$, the presample aperture was at about $22 \mathrm{~m}$ from the source, the sample stage was at $26 \mathrm{~cm}$ from the aperture and the analyzer and detector a further $2.5 \mathrm{~m}$ downstream. The detector was a CCD (Photonic Science, UK) with a pixel size of $12.5 \mu \mathrm{m}$.

Five terms were retained for the IF $(N=5)$ and the scattering distribution was assumed to be Gaussian $(M=1)$. The three parameters representing absorption, refraction, and scattering were obtained by a pixel-wise nonlinear fit [30] comparing the intensities recorded with and without the sample. For image acquisition, the IF was sampled in five positions with $\{ \pm 24, \pm 12,0\} \mu$ m displacement with respect to the position of maximum intensity in the central slit of the analyzer, with an exposure of $400 \mathrm{~ms}$ each.

The phantom used for planar imaging was composed of an acrylic cylinder, density $1.18 \mathrm{~g} / \mathrm{cm}^{3}$ and radius
$1.45 \mathrm{~mm}$, and a step wedge made of paper. Paper was chosen because it contains features on a wide range of scales, extending to submicron [37]. A melamine sponge prism was used to experimentally measure the dependency of the darkfield signal upon thickness. Monodisperse borosilicate microspheres with diameters of 5,10 , and $12 \mu \mathrm{m}$ were embedded into an acrylic support for CT acquisitions. A plastic scaffold was used to test the three-dimensional reconstruction on a phantom with a more complex geometry.

The data were recorded at a pixel size of $12.5 \mu \mathrm{m}$ and subsequently binned in such a way that the integrated intensity going through a single aperture in the analyzer was combined in a single image line. In radiography image mode, the sample was scanned along $y$ in $15 \mu \mathrm{m}$ steps to build up a two-dimensional image. 600 views were acquired with $0.3^{\circ}$ angular step for CT.

The experimental IF is compared to a numerical simulation [38] that incorporates all the experimental parameters, with a good match that can be seen in Fig. 1(b). This IF is taken as the reference one, and subsequently used for the retrieval of the sample images. The approximate rocking curve of a $\mathrm{Si}$ (111) crystal at the energy of $20 \mathrm{keV}$ is also plotted in Fig. 1(b), for a visual comparison of the sensitivity curves of the EI and the analyzer crystal based imaging methods. It is interesting to note that the EI configuration reported here offers comparable curve width to that of the crystal analyzer, at least when the (111) diffraction is exploited in the latter.

In order to investigate the dependence of the retrieved signals on the spatial resolution, data were analyzed two
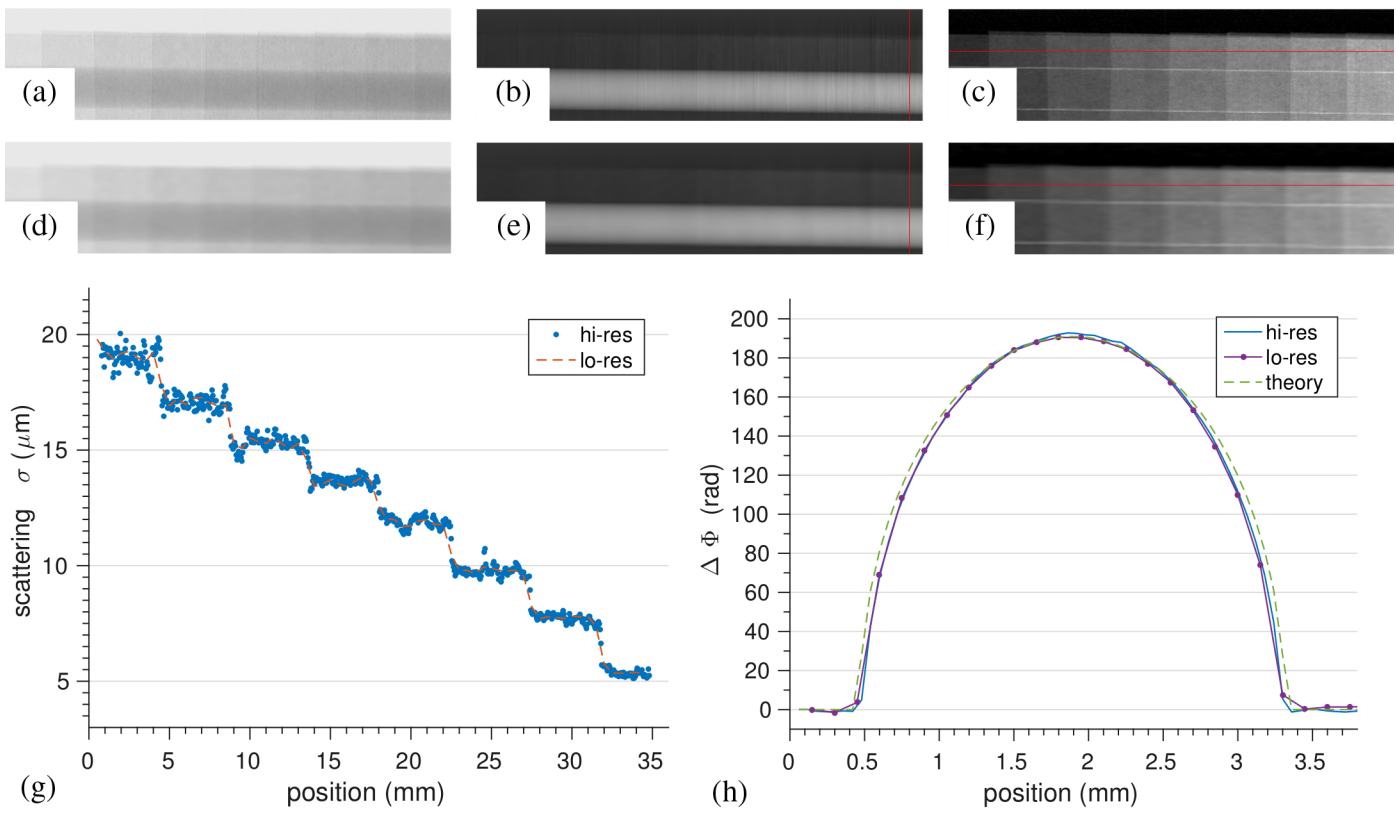

FIG. 2. Phantom consisting of a perspex cylinder and a paper step wedge: transmission (a) and (d), phase (b) and (e), and dark field (c) and (f). The images at high resolution, in panels (a)-(c), can be compared to the images at low resolution, shown in panels (d)-(f). The profiles reporting the dark-field signal (g) and the phase shift (h) measured along the lines highlighted in the panels (c),(f) and (b),(e), respectively, show that the spatial resolution does not affect accuracy. A very good match between theoretically expected and experimentally measured profiles can be observed for the phase image. 

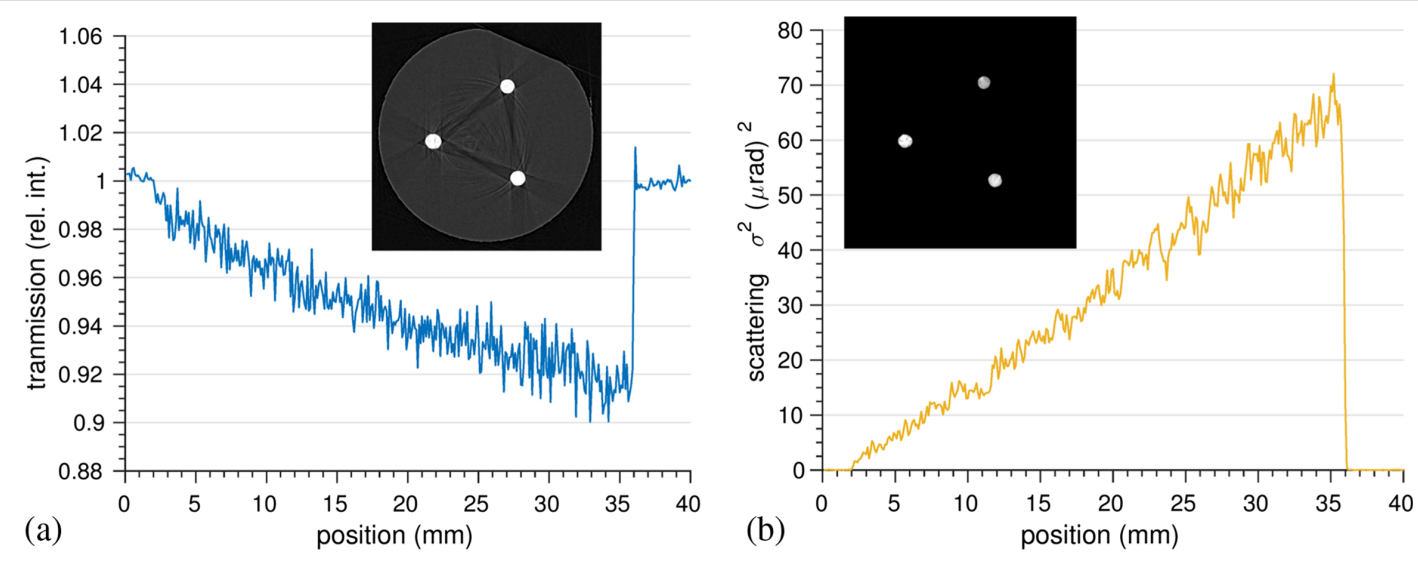

FIG. 3. Quantitative profiles of transmission (a) and dark field (b) extracted from images of the melamine sample. The dark-field signal $\sigma^{2}$ is directly proportional to the sample thickness, as expected. The two insets show CT slices absorption (a) and dark-field (b) images of the microspheres embedded in acrylic.

times. In the first case (high resolution) the pixel size was $50 \mu \mathrm{m} \times 60 \mu \mathrm{m}$ while in the second case (low resolution) the pixel size was $500 \mu \mathrm{m} \times 150 \mu \mathrm{m}$. These two sets of data were obtained by binning the raw intensity acquisitions, after which the same analysis procedure is applied to both data sets. The results of this analysis are summarized in Fig. 2. The effect of lowering the spatial resolution can be observed by comparing panels (a)-(c) to panels (d)-(f) in Fig. 2, where the high- and low-resolution images are reported, respectively. For a quantitative comparison, line profiles are shown in the (g) and (h) panels of Fig. 2. They were extracted along the directions highlighted in panels (c),(f) for the plots in (g) and in panels (b),(e) for the plots in (h). They represent the dark-field signal measured along the paper step wedge and the phase signal measured across the plastic cylinder. The values obtained in the high- and lowresolution configurations are quantitatively very close to each other. Moreover, the theoretical phase shift shows a

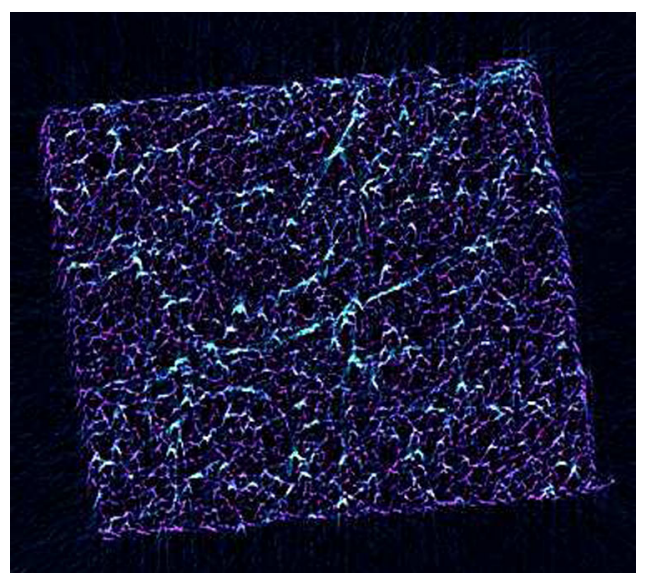

FIG. 4. Absorption and dark-field CT of a scaffold in false colors (absorption: magenta and dark field: cyan), blended. It can be seen how the two different contrast channels provide a complementary representation of this complex sample. very good match with the experimentally measured one $\left(\delta=6.61 \times 10^{-7}\right.$ and $\beta=3.34 \times 10^{-10}$ [39]), even though the experimental profiles in Fig. 2(h) were extracted from the portion of the cylinder placed behind the part of the wedge with strongest scattering. The dispersion of the retrieved refraction angles, measured in an empty background region, can be used to estimate the sensitivity of the imaging system $[27,28,40]$. By following this procedure, the high and low spatial resolution configurations gave a standard deviation of 17 and 5.9 nrad, respectively. The behavior of transmission and dark-field signal as a function of thickness were investigated by means of the melamine sponge. Two line plots are reported in panels (a) and (b) of Fig. 3. The dark-field signal grows linearly with the sample thickness, and the expected exponential relationship holds for the transmission. The dark-field CT result for the microspheres sample is shown in the inset of Fig. 3(b), along with the standard absorption CT image again showed as an inset in Fig. 3(a). The values $\{0.95 \pm 0.06,1.10 \pm$ $0.05,1.19 \pm 0.08\} \times 10^{3} \mu \mathrm{rad}^{2} \mathrm{~mm}^{-1}$ were measured for the $\{5,10,12\} \mu \mathrm{m}$ diameter spheres details, respectively. Finally, as an example with a more complex geometry, the image obtained by fusing absorption- and dark-field- CT reconstructions of a plastic scaffold is shown in Fig. 4. The two color channels emerge from different sample details, meaning that they represent different properties of the sample and can offer a complementary visualization of its characteristics.

In conclusion, we presented a multiaperture analyzer setup for x-ray phase-contrast radiography and computed tomography. The linearity of the dark-field signal with the sample thickness was experimentally verified and quantitative computed tomography was performed on a custom phantom made of microspheres embedded into an acrylic support. The method simultaneously provides absorption, refraction, and dark-field contrasts. It is based on edge illumination and it is anticipated that the negligible coherence requirements and the achromatic properties of this 
method will be useful for its translation into laboratorybased instruments. Very good agreement was observed between theory and experiment for the phase-shift image of a test sample. Notably, the phase shift was quantitatively measured to a high degree of accuracy also in the presence of strong scatterers, showing the simultaneous attainment of high sensitivity and dynamic range. Finally, as an example of a complex geometry sample, the images of a scaffold were presented.

This project was supported by the UK Engineering and Physical Sciences Research Council Grant No. EP/ I021884/1. M. E. was supported by the Royal Academy of Engineering under the RAEng Research Fellowships scheme. F. A. V. is supported by the EPSRC (Grant No. EP/ M507970/1). We thank Elettra Sincrotrone Trieste for access to SYRMEP beam line (Proposal No. 20140147) that contributed to the results presented here.

*m.endrizzi@ucl.ac.uk

[1] A. Bravin, P. Coan, and P. Suortti, Phys. Med. Biol. 58, R1 (2013).

[2] S. Wilkins, Y. I. Nesterets, T. Gureyev, S. Mayo, A. Pogany, and A. Stevenson, Phil. Trans. R. Soc. A 372, 0021 (2014).

[3] U. Bonse and M. Hart, Appl. Phys. Lett. 6, 155 (1965).

[4] K. Goetz, M. P. Kalashnikov, Y. A. Mikhaŭlov, G. V. Sklizkov, S. I. Fedotov, E. Foerster, and P. Zaumseil, Sov. J. Quantum Electron. 9, 607 (1979).

[5] T. J. Davis, D. Gao, T. E. Gureyev, A. W. Stevenson, and S. W. Wilkins, Nature (London) 373, 595 (1995).

[6] V. N. Ingal and E. A. Beliaevskaya, J. Phys. D 28, 2314 (1995).

[7] S. W. Wilkins, T. E. Gureyev, D. Gao, A. Pogany, and A. W. Stevenson, Nature (London) 384, 335 (1996).

[8] D. Chapman, W. Thomlinson, R. E. Johnston, D. Washburn, E. Pisano, N. Gmür, Z. Zhong, R. Menk, F. Arfelli, and D. Sayers, Phys. Med. Biol. 42, 2015 (1997).

[9] J. F. Clauser, U. S. Patent 5,812,629 (1998).

[10] C. David, B. Nohammer, H. H. Solak, and E. Ziegler, Appl. Phys. Lett. 81, 3287 (2002).

[11] A. Momose, S. Kawamoto, I. Koyama, Y. Hamaishi, K. Takai, and Y. Suzuki, Jpn. J. Appl. Phys. 42, L866 (2003).

[12] S. C. Mayo and B. Sexton, Opt. Lett. 29, 866 (2004).

[13] F. Pfeiffer, T. Weitkamp, O. Bunk, and C. David, Nat. Phys. 2, 258 (2006).

[14] M. D. de Jonge, B. Hornberger, C. Holzner, D. Legnini, D. Paterson, I. McNulty, C. Jacobsen, and S. Vogt, Phys. Rev. Lett. 100, 163902 (2008).

[15] H. Wen, E. E. Bennett, M. M. Hegedus, and S. Rapacchi, Radiology 251, 910 (2009).

[16] K. S. Morgan, D. M. Paganin, and K. K. W. Siu, Appl. Phys. Lett. 100, 124102 (2012).

[17] H. Wang, Y. Kashyap, and K. Sawhney, Phys. Rev. Lett. 114, 103901 (2015)

[18] H. Miao, A. Panna, A. A. Gomella, E. E. Bennett, S. Znati, L. Chen, and H. Wen, Nat. Phys. 12, 830 (2016).
[19] A. Olivo, F. Arfelli, G. Cantatore, R. Longo, R. H. Menk, S. Pani, M. Prest, P. Poropat, L. Rigon, G. Tromba, E. Vallazza, and E. Castelli, Med. Phys. 28, 1610 (2001).

[20] P. R. Munro, K. Ignatyev, R. D. Speller, and A. Olivo, Proc. Natl. Acad. Sci. U.S.A. 109, 13922 (2012).

[21] M. Endrizzi, P. C. Diemoz, T. P. Millard, J. L. Jones, R. D. Speller, I. K. Robinson, and A. Olivo, Appl. Phys. Lett. 104, 024106 (2014).

[22] A. Olivo and R. Speller, Appl. Phys. Lett. 91, 074106 (2007).

[23] M. Endrizzi, F. A. Vittoria, P. C. Diemoz, R. Lorenzo, R. D. Speller, U. H. Wagner, C. Rau, I. K. Robinson, and A. Olivo, Opt. Lett. 39, 3332 (2014).

[24] P. R. T. Munro, K. Ignatyev, R. D. Speller, and A. Olivo, Opt. Express 18, 19681 (2010).

[25] M. Endrizzi, F. A. Vittoria, G. Kallon, D. Basta, P. C. Diemoz, A. Vincenzi, P. Delogu, R. Bellazzini, and A. Olivo, Opt. Express 23, 16473 (2015).

[26] M. Marenzana, C. K. Hagen, P. D. N. Borges, M. Endrizzi, M. B. Szafraniec, K. Ignatyev, and A. Olivo, Phys. Med. Biol. 57, 8173 (2012).

[27] P. C. Diemoz, M. Endrizzi, C. E. Zapata, Z. D. Pešić, C. Rau, A. Bravin, I. K. Robinson, and A. Olivo, Phys. Rev. Lett. 110, 138105 (2013).

[28] P. Diemoz, C. Hagen, M. Endrizzi, and A. Olivo, Appl. Phys. Lett. 103, 244104 (2013).

[29] T. P. Millard, M. Endrizzi, K. Ignatyev, C. K. Hagen, P. R. T. Munro, R. D. Speller, and A. Olivo, Rev. Sci. Instrum. 84, 083702 (2013).

[30] M. Endrizzi, D. Basta, and A. Olivo, Appl. Phys. Lett. 107, 124103 (2015)

[31] A. Olivo, S. Gkoumas, M. Endrizzi, C. K. Hagen, M. B. Szafraniec, P. C. Diemoz, P. R. T. Munro, K. Ignatyev, B. Johnson, J. A. Horrocks, S. J. Vinnicombe, J. L. Jones, and R. D. Speller, Med. Phys. 40, 090701 (2013).

[32] C. Hagen, P. Munro, M. Endrizzi, P. Diemoz, and A. Olivo, Med. Phys. 41, 070701 (2014).

[33] L. Rigon, A. Astolfo, F. Arfelli, and R.-H. Menk, European Journal of Radiology 68, S3 (2008).

[34] Z.-T. Wang, K.-J. Kang, Z.-F. Huang, and Z.-Q. Chen, Appl. Phys. Lett. 95, 094105 (2009).

[35] M. Bech, O. Bunk, T. Donath, R. Feidenhans, C. David, and F. Pfeiffer, Phys. Med. Biol. 55, 5529 (2010).

[36] F. A. Vittoria, M. Endrizzi, P. C. Diemoz, A. Zamir, U. H. Wagner, C. Rau, I. K. Robinson, and A. Olivo, Sci. Rep. 5, 16318 (2015).

[37] See Supplemental Material at http://link.aps.org/ supplemental/10.1103/PhysRevLett.118.243902 for scanning electron microscope characterization of the material, as well as for a comparison of the performance of the multiaperture against the single-aperture analyzer.

[38] F. A. Vittoria, P. C. Diemoz, M. Endrizzi, L. Rigon, F. C. Lopez, D. Dreossi, P. R. T. Munro, and A. Olivo, Appl. Opt. 52, 6940 (2013).

[39] CSIRO CSS eToolbox, https://www.ts-imaging.net.

[40] P. Modregger, B. R. Pinzer, T. Thüring, S. Rutishauser, C. David, and M. Stampanoni, Opt. Express 19, 18324 (2011). 